\title{
Perfil profesional de los trabajadores del sector del fitness en la ciudad de Zaragoza Professional profile of workers in the fitness sector in the city of Zaragoza
}

Nerea Estrada-Marcén, Gonzalo Sanz Gonzalo, Jaime Casterad Seral, Javier Simón Grima, Alberto Roso Moliner

\author{
Universidad de Zaragoza (España)
}

\begin{abstract}
Resumen: Actualmente el sector del Fitness se encuentra en auge en nuestro país, lo que acarrea un incremento importante de los puestos de trabajo relacionados con él. Sin embargo, el perfil de sus trabajadores apenas ha sido estudiado. La finalidad de este trabajo es describir las características de los profesionales empleados en centros de Fitness en una ciudad representativa a nivel nacional como es Zaragoza (España). Dicha descripción la realizan los Directores técnicos y/o coordinadores de los centros contratantes. En el sector del consumo la capital aragonesa suele ser la urbe que mejor representa la realidad socioeconómica española, puesto que tiene un tamaño grande, situándose a medio camino entre Madrid, Barcelona y Euskadi. Zaragoza reúne las condiciones para representar bien a la media española en muchos estudios de mercado. Participaron en el estudio 19 centros, y sus directores técnicos o coordinadores cumplimentaron un cuestionario creado al efecto. Los porcentajes de hombres y mujeres que trabajan en el sector en la ciudad de Zaragoza están muy igualados. Los centros de trabajo suelen establecer políticas de incentivación de la formación continua, compartiendo sus gastos frecuentemente entre trabajador y empresa (79\%). La gran mayoría de los centros cuenta al menos con un egresado en Ciencias de la Actividad Física y del Deporte, que realiza labores de monitor o instructor en la mayoría de casos. Ala hora de contratar a los profesionales de un centro de fitness, prima la experiencia laboral por delante de las competencias en el puesto, la personalidad, o la titulación, en este orden.
\end{abstract}

Palabras clave: Gestión Deportiva, Profesionales, Fitness, Entrenamiento, Cualificaciones, Experiencia Profesional.

Abstract: Currently, the Fitness sector is booming in our country, which leads to a significant increase in jobs related to it. However, the profile of its workers has barely been studied. The purpose of this work is to describe the characteristics of professionals employed in fitness centers in a representative city at a national level such as Zaragoza (Spain). Said description is made by the Technical Directors and/or coordinators of the contracting centers. In the consumer sector the Aragonese capital is usually the city that best represents the Spanish socioeconomic reality, since it has a large size, located halfway between Madrid, Barcelona and Euskadi. Zaragoza meets the conditions to represent well the Spanish average in many market studies. 19 centers participated in the study and their technical directors or coordinators completed a questionnaire created for this purpose. The percentages of men and women working in the sector in the city of Zaragoza are very close. The work centers usually establish incentive policies of continuous training, sharing their expenses frequently between worker and company (79\%). The vast majority of centers have at least one graduate in Physical Activity and Sports Sciences, who performs tasks of monitorer or instructor in most cases. When it comes to hiring professionals of a fitness center, work experience prevails in front of the skills in the position, personality, or education degree, respectively. Keywords: Sports Management, Professionals, Fitness, Training, Qualifications, Professional Experience.

\section{Introducción}

Las actividades de fitness en nuestro país se encuentran en la actualidad en auge. En los últimos años ha aumentado el número de clientes adscritos a los centros españoles, casi triplicando la cifra de 2006, obteniendo un total de 6.400 .000 socios, colocando a España en el quinto lugar a nivel mundial en este aspecto, y cuarto a nivel continental según la International Health \& Racquet Sports Association (IHRSA, 2013). Anivel europeo, España en los últimos tiempos ha sido la tercera potencia del fitness en cuanto a ingresos producidos, habiendo subido un puesto desde 2006, siendo la sexta industria del fitness mundial en cuanto a ingresos generados, y estando en el décimo puesto en cuanto a número de centros en funcionamiento (IHRSA, 2013). De forma paralela, ha ido aumentando exponencialmente en nuestro país la demanda específica de productos vinculados con la actividad física y la salud (Martínez, González, Jiménez-Beatty, Graupera, Martín, Campos y Del Hierro, 2009), y la oferta de programas y actividades orientadas hacia la salud en los centros de fitness españoles ha ido creciendo y diversificándose (Tracogna y Rial-Rebullido, 2018).

Un auge como el mencionado de las actividades de Fitness en nuestro país, acarrea un incremento importante de los puestos de trabajo relacionados en los últimos años (Instituto Nacional de las Cualificaciones-Consejo Superior de Deportes, 2008). España tiene características particulares respecto al tipo de instalaciones y equipamientos utilizados, a las actividades y servicios ofertados y a las características de los profesionales que trabajan en este sector (IHRSA, 2007). En relación a este último aspecto, los costes en recursos humanos representan de media un 32,9\% de los ingresos totales del sector

Fecha recepción: 15-03-17. Fecha de aceptación: 19-07-18 Nerea Estrada-Marcén

nereaes@unizar.es deportivo según el Informe global de las instalaciones deportivas españolas (Consultora Management Around Sports, 2014). Se han diversificado las profesiones vinculadas con el fitness, y se han generado varios empleos relacionados con este sector (CDS, 2000), viéndose aumentado en gran medida el número de profesionales que trabajan en centros de fitness (Boned, Felipe, Barranco, Grimaldi-Puyana, y Crovetto, 2015). Sin embargo, en la mayoría de las Comunidades Autónomas las profesiones relacionadas con el sector estaban aún sin regular a comienzos de década (García, Lago y Fernández, 2011), y lo siguen estando en la actualidad.

A pesar de estos datos boyantes, la situación de la industria del fitness en España ha sido escasamente estudiada, siendo la mayoría de los trabajos realizados en este ámbito estudios de mercado (Lagrosen y Lagrosen, 2007). Y aunque aspectos como la imagen de las instalaciones y su equipamiento han sido tradicionalmente más valorados y mimados en el sector del fitness que los profesionales contratados en dicha instalación (Boned, et al., 2015), parece que esta tendencia está cambiando. La gran competitividad del sector hace que los recursos humanos sean un aspecto fundamental a la hora de diferenciarse del resto de centros (Gambau, 2011). Este aspecto cobra gran importancia puesto que la labor de los profesionales en un centro de fitness es un aspecto fundamental para su buen funcionamiento, encontrándonos ante un factor clave en la retención de usuarios (IHRSA, 2011).

Pero, ¿hay un perfil claro de profesional de las actividades de Fitness?, ¿cómo es la situación laboral de los trabajadores del sector?. Consideramos de interés tratar de dar respuesta a estas preguntas describiendo la situación actual de dichos trabajadores, lo que nos permitirá entender la realidad de una profesión en auge, y conocer aquellos aspectos susceptibles de cambios en los que se pueda incidir para implementar mejoras.

Objetivo: Describir el perfil profesional de los trabajadores de centros de fitness de carácter público y privado en la ciudad de Zaragoza, recabando para ello información de los contratadores. 


\section{Metodología}

\section{Participantes}

La población de estudio son los directores técnicos o coordinadores de los centros ofertantes de actividades de Fitness de la ciudad de Zaragoza, tanto de carácter público como privado, que contasen al menos con sala de musculación y con sala de actividades dirigidas. Ellos responderían sobre la situación específica de los trabajadores de sus centros.

Determinar el universo de estudio ha resultado complicado, ante la imposibilidad de encontrar un censo específico de centros de Fitness. Se trató de obtener un listado en el Registro deAsociaciones Deportivas de Aragón, en el Registro Mercantil y en la Cámara de Comercio e Industria de Zaragoza; ninguna de dichas instituciones podía ofrecernos una lista en la que se encontrasen censadas las empresas de este ámbito. El censo de instalaciones del Consejo Superior de deportes (2007) tampoco lograba ofrecer resultados satisfactorios a una búsqueda tan específica.

Además, los puestos de trabajo referidos al mercado de trabajo de la actividad física y del deporte no se recogen estadísticamente de forma específica y por ello es difícil conocer exactamente su cuantificación, y han de deducirse de diversos cálculos, encuestas y estadísticas (Campos, 2012).

De este modo, se optó por elaborar una base de datos a través de la búsqueda por internet, empleando buscadores generales y específicos del ámbito del Fitness, tratando de recoger la totalidad o casi la totalidad de centros de la ciudad de Zaragoza. Se obtuvo de esta forma un listado previo del que hubo que eliminar las coincidencias, así como los centros no existentes o que habían cambiado de nombre, y se concretó la dirección, código postal y número de teléfono del centro, obteniendo así un universo de 176 centros. Las fuentes consultadas para la elaboración de

\begin{tabular}{|c|c|}
\hline Fuente & Resultados \\
\hline Páginas Amarillas de Zaragoza & 70 \\
\hline Fitnesszona.com & 77 \\
\hline Hispagimnasios.com & 80 \\
\hline Musculacion.net & 62 \\
\hline Revista Sport Life & 19 \\
\hline Guiasamarillas.es & 93 \\
\hline Buscador de Google maps & 87 \\
\hline Buscagym.com & 50 \\
\hline Gimnasios.net & 69 \\
\hline
\end{tabular}

la base de datos quedan reflejadas en la tabla 1.

El siguiente paso fue comprobar a través de la página web de cada centro los servicios ofertados. En caso de no tener página web, se procedió a llamar por teléfono. Se eliminaron los centros que no disponían al menos de una sala de fitness o musculación, y de una sala de actividades dirigidas con más de una actividad diferente. Los centros desestimados eran principalmente centros especializados dedicados a las Artes Marciales, deportes de combate, Pilates, o que únicamente contaban con sala de musculación. Finalmente se estableció el universo de estudio en 54 centros, de los cuales 33 ofrecían servicio de entrenamiento personal.

Se contactó vía telefónica con la totalidad de centros para informar sobre los objetivos y procedimiento de nuestro estudio, y solicitando una vía de contacto electrónica con el coordinador/gerente/director. De ese modo se les facilitaría el enlace al cuestionario correspondiente en caso de querer participar. Tras esta llamada, 5 centros (9\%) rehusaron participar, de modo que 49 centros recibieron vía correo electrónico el cuestionario. Finalmente, la muestra estuvo compuesta por 19 directores técnicos/coordinadores de centros de Fitness, que fueron los que cumplimentaron la encuesta, a pesar de que un $91 \%$ del universo de estudio había recibido el cuestionario tras acceder a participar.

\section{Instrumento}

El cuestionario, creado ad hoc a partir del de García (2011), estaba compuesto por cuatro apartados claramente diferenciados: Información general, servicios ofrecidos, profesionales que trabajan en la instalación y equipamientos e instalaciones; en este artículo se presentan los resultados relacionados con el apartado «Profesionales que trabajan en la instalación». En dicho apartado se combinaban preguntas con opciones de respuesta abierta y cerrada, según se considerase más oportuno en cada caso.

De esta forma se trataba de recabar información acerca de las características de los puestos de trabajo del centro, de los trabajadores contratados, y de las cualidades valoradas por los contratadores en el puesto de trabajo.

\section{Procedimiento}

Para la aplicación de este cuestionario se utilizó la herramienta «Google Drive». Se envió un enlace online a través del correo electrónico, junto con información sobre el estudio, a cada uno de los centros que accedieron a participar. A los 10 días se envió otro correo electrónico como recordatorio.

\section{Análisis de los datos}

Para el análisis estadístico se ha realizado un análisis descriptivo a través del paquete estadístico SPSS, versión 19.0. Se calcularon así los valores de media, desviación típica, máximo, mínimo y porcentajes, según la naturaleza de los datos.

\section{Resultados y Discusión}

\section{Género de los trabajadores}

Tal como se refleja en la figura 1, el 55\% de los puestos de trabajo están ocupados por hombres, mientras que el $45 \%$ restante los ocupan mujeres. Esta ligera superioridad en cuanto a la presencia del género masculino, concuerda con otros estudios; parece que los contratos dentro del ámbito de la actividad física y del deporte en España son firmados mayoritariamente por hombres. Nuestros datos van en la línea de los del estudio de Campos (2006), donde se afirma que dentro de los empleos propios de la actividad física y en deporte, un 58,5\% están cubiertos por hombres. En el trabajo de Boned et al. (2015), que trata de describir el perfil de los trabajadores de los centros de fitness en España, encontramos unos porcentajes aún más igualados, puesto que el 50,7\% de los participantes en su estudio son hombres, frente a un $49,3 \%$ de mujeres.

En el trabajo de Alves, da Costa, Castañer, Fernández, y Anguera (2013), donde se analiza la conducta de los instructores de Fitness, la presencia de hombres en la muestra es ligeramente superior (51.6\%) a la de mujeres. Y en el estudio de Juan-Llamas (2015), la presencia de hombres y mujeres en la muestra, compuesta por instructores de clases colectivas, es prácticamente igual (49,5\% de hombres frente a 50,5\% de mujeres). Puede que en estos dos casos se haya buscado intencionalmente obtener estos porcentajes tan equitativos, para reflejar la realidad del sector o por cualquier otro motivo.

A pesar de que todo apunta a que los hombres tienen una mayor presencia que las mujeres en este sector profesional, parece que hoy por hoy las cifras están muy igualadas.

\section{Tipo de contrato de los trabajadores}

Atendiendo al tipo de contrato, en la figura 2 podemos observar cómo el 9\% de los trabajadores pertenecen a una empresa externa o son autónomos, mientras que el $91 \%$ de los profesionales que encontramos en los centros de fitness participantes en nuestro estudio son contratados por la propia empresa.

Un estudio de Pérez, Vilanova y Grimaldi-Puyana (2016), centrado de forma específica en el mercado actual de trabajo de los graduados en ciencias de la actividad física y el deporte, informa de que el mantenimiento y la salud es el cuarto ámbito de inserción del egresado en la titulación mencionada, ofreciendo unas condiciones con una clara tendencia a la flexibilidad laboral. Según este trabajo, el $29 \%$ de las ocupaciones de los graduados en el ámbito del mantenimiento físico y de la salud se desarrollan mediante la fórmula de autónomos. Los yacimientos laborales vinculados con el mantenimiento y la salud, y con la gestión en el ámbito privado, ya sea en entidades con o sin ánimo de 
lucro, son los que presentan un mayor nivel de ocupaciones mediante la fórmula de autónomos en el caso concreto de estos titulados universitarios (Pérez, et al., 2016). En cualquier caso, nuestro estudio y el de Pérez et al. reflejan poblaciones distintas.

\section{Tipo de dedicación de los trabajadores}

En la figura 3 se refleja cómo el 58\% de los trabajadores de los centros participantes están contratados a tiempo parcial frente a un $42 \%$ contratado a tiempo completo. En este sentido, hay varios estudios con resultados parecidos, afirmando que el tipo de contrato más frecuente en los servicios de actividad física y deporte es el parcial (Campos, 2000; INCUAL, 2008).

Parece que la temporalidad también es una característica destacable del mercado laboral de la actividad física y del deporte, habiendo una elevada contratación temporal en la mayoría de los centros e instalaciones deportivas (Campos, 2006). Y la precariedad también parece ser un sello de identidad de los empleos vinculados a la actividad físico-deportiva. En este sentido, nos parecen reseñables los datos del trabajo de Gallardo y Campos (2011), que al estudiar concretamente a trabajadores del ámbito profesional de la actividad física y deportiva, observan que el 56,3\% de ellos tienen retribución económica pero no contrato; y de los trabajadores con contrato, únicamente un 20,4\% tienen un contrato laboral indefinido. Aunque nuestro estudio no arroja datos sobre la temporalidad o sobre la existencia de vínculo contractual, podría ser interesante contemplar estos aspectos en posteriores trabajos.

Parece que los trabajadores con mayor nivel formativo en el ámbito de la actividad física y deportiva, los egresados en Ciencias de la Actividad Física y del Deporte (CCAFD), se caracterizan también por su multiocupación, encadenando contratos parciales (Pérez, et al., 2016). Un $38 \%$ de ellos desarrollan más de un trabajo en el ámbito deportivo. La media de ocupaciones disminuye a medida que las promociones son más veteranas y se incrementa en las últimas promociones, lo que según Pérez et al. (2016) podría deberse a dos motivos principales: imposibilidad de encontrar un trabajo a tiempo completo y con una remuneración que se considere suficiente para vivir, y tendencia a la flexibilización del mercado de trabajo, consistente en la ampliación de las modalidades de contratación y en el abaratamiento del despido. Por otra parte, Boned, et al. (2015) reflejan en su estudio que el 13.6\% de los profesionales del fitness combina simultáneamente estudios universitarios con su trabajo, lo que podría justificar también que un porcentaje de trabajadores busque de forma voluntaria un contrato parcial que les permita compatibilizar empleo y formación.

Aunque es complicado discernir las causas, parece en todo caso que la contratación parcial es generalizada en el mercado laboral de la actividad física y deportiva.

\section{Política de formación continua}

El 77,8\% de los centros participantes en nuestro estudio tenía establecida alguna política de formación continua para sus trabajadores. En la figura 4 se aprecian los tipos de formación continua que llevaban a cabo los centros zaragozanos.

La mayoría de los centros, para llevar a cabo los planes de formación continua, favorecen la realización de cursos ajenos a la empresa que suelen suscitar el interés por parte de los trabajadores (64,3\%). En menor medida se organizan cursos en el propio centro $(57,1 \%)$, o encargan la oferta de los cursos a entidades externas (28,6\%); un 35,7\% organiza salidas de sus trabajadores a congresos, ferias, o eventos del sector, y un 21,4\% de los centros cuentan con documentación actualizada y especializada a disposición de sus trabajadores (libros, revistas, etc).

Atendiendo a los resultados de Telecyl Estudios (2006) sobre el sector de la actividad física a nivel general (no únicamente fitness) dentro del panorama nacional, el 21,3\% de técnicos en este ámbito recibía planes de formación continua por parte de sus empresas. El estudio de García (2011), centrado en el sector del fitness, obtuvo que un 55,9\% de centros en Madrid realizaban planes de formación continua. En todo caso, los resultados arrojados en nuestro estudio reflejan una gran pre-

ocupación de los centros de Fitness de la ciudad de Zaragoza por la formación continua y la actualización de sus trabajadores.

Sería interesante comprobar si este interés de los centros por formar a los trabajadores va acompañado por una respuesta positiva de éstos hacia dicha actualización, como parece lógico suponer. En el estudio de Juan-Llamas (2015), centrado en los instructores de clases colectivas del sector del fitness, un 56,7\% de los consultados declararon tomar parte en cursos de una a tres veces al año o más de tres veces al año, y un 46,8\% de estos encuestados, asistían a seminarios, eventos o convenciones en el mismo período. Un trabajo de Portes-Junior, Lagos, Domínguez-Riquelme y López-López (2013) menciona que las exigencias laborales del trabajo en los centros deportivos tiene un carácter multidisciplinar, y el trabajo de Juan-Llamas (2015) afirma que un 85,6\% de los instructores de fitness realizan más de un tipo de clases colectivas, lo que parece evidenciar la necesidad del profesional del sector de recibir formación de forma continuada para estar actualizado, y poder así adaptarse a las exigencias del mercado laboral. Se trata además de un sector en constante cambio, donde el reciclaje parece convertirse en una obligación. Sería interesante en trabajos futuros analizar la respuesta de los trabajadores hacia la formación continua y las motivaciones para su realización.

En la figura 5 se aprecia que los gastos de formación continua se comparten entre la empresa y el trabajador en el 79\% de los centros, y en el $21 \%$ de los casos corren siempre a cargo de la empresa.

\begin{tabular}{|c|c|}
\hline+2 & $\begin{array}{l}55 \% \\
45 \% \\
\end{array}$ \\
\hline \multicolumn{2}{|c|}{ Figura 2. Trabajadores contratados por el centro según tipo de contrato } \\
\hline $\begin{array}{l}\text { Trabajadores propios } \\
\text { Autónomos o contratados por empresa externa }\end{array}$ & $\begin{array}{l}91 \% \\
9 \%\end{array}$ \\
\hline \multicolumn{2}{|c|}{ Figura 3. Tipo de dedicación de los trabajadores de los centros } \\
\hline Dedicación completa & $42 \%$ \\
\hline Dedicación parcial & $58 \%$ \\
\hline \multicolumn{2}{|c|}{ Figura 4. Planes de formación continua en los centros de fitness de Zaragoza } \\
\hline Favorece realización cursos ajenos empresa & $64 \%$ \\
\hline Organiza cursos en propio centro & $57 \%$ \\
\hline Encargan oferta entidades externas & $29 \%$ \\
\hline Organiza salidas a congresos, ferias, eventos... & $36 \%$ \\
\hline El centro tiene documentación especializada & $21 \%$ \\
\hline \multicolumn{2}{|c|}{ Figura 5. Responsabilidad de los costes de la formación continua } \\
\hline Siempre empresa & $21 \%$ \\
\hline Compartidos empresa y trabajador & $79 \%$ \\
\hline \multicolumn{2}{|c|}{ Figura 6. Disponibilidad de catálogo de funciones y características de cada puesto } \\
\hline Sí & $54 \%$ \\
\hline No & $46 \%$ \\
\hline \multicolumn{2}{|c|}{ Figura 7. Presencia del Licenciado/Graduado en CCAFD en el centro } \\
\hline$\overline{\text { Sí }}$ & $82 \%$ \\
\hline No & $18 \%$ \\
\hline \multicolumn{2}{|c|}{$\begin{array}{l}\text { Figura 8. Distribución de los Licenciados/Graduados en Ciencias de la Actividad Física y de } \\
\text { Deporte presentes en los centros de fitness de Zaragoza }\end{array}$} \\
\hline Gerencia/dirección & $18 \%$ \\
\hline Dirección técnica & $23 \%$ \\
\hline Recepción & $8 \%$ \\
\hline Entrenamiento personal & $15 \%$ \\
\hline Monitor sala o act. Dirigidas & $36 \%$ \\
\hline
\end{tabular}

Figura 9. Valoración por parte de los contratadores de las "competencias y/o habilidades en el puesto"

Poco importante

Importante

Bastante importante $11 \%$ Muy importante $50 \%$ $11 \%$

Figura 10. Valoración por parte de los contratadores de la "formación" Poco importante

Importante

Bastante importante

Muy importante

$25 \%$

$19 \%$

Figura 11. Valoración por parte de los contratadores de la "personalidad" Poco importante

Importante

Bastante importante

Muy importante 


\section{Catálogo de funciones y características de los puestos}

Conocer cuáles son las profesiones del deporte implica una labor de búsqueda complicada puesto que no existe uniformidad en su identificación(Gambau, 2011).

Según se refleja en la figura 6, el 56,3\% de los centros de fitness participantes poseen un catálogo que describe las funciones y características de cada puesto de trabajo ocupado por sus empleados. García (2011) encontró en su estudio un 32,2\% de centros que poseían este catálogo de funciones de los empleados. Parece una tendencia en auge la de establecer protocolos en los centros de fitness para asegurar que los instructores mantengan una calidad homogénea en sus servicios.

Es necesario señalar que estudios como el de Nuviala, Tamayo, Iranzo, y Falcón (2008) citan los recursos humanos como uno de los puntos fuertes en la satisfacción de los clientes en los centros deportivos, y parece que la conducta del instructor de fitness influye de forma directa en la satisfacción manifestada por los usuarios de dichos centros (Alves et al., 2013). Parece que una mejor actuación de los instructores en sus sesiones puede ayudar a aumentar los niveles de satisfacción de los practicantes (DoYoung, Hyungil, Teck y CheeKeng, 2010), lo que puede facilitar la adhesión a la práctica de ejercicio físico y la fidelización (Lippke, Knäuper y Fuchs, 2003; Loughead y Carron, 2004; Loughead, Colman y Carron, 2001). A tenor de ello, vemos fundamental que los centros busquen crear protocolos que logren y permitan mantener un servicio de calidad de sus trabajadores.

Presencia de licenciados/graduados en Ciencias de la actividad físicay del deporte en los centros

Por lo que respecta a la contratación de licenciados/graduados en CCAFD en los centros de fitness participantes, observamos en la figura 7 cómo el 82,4\% de ellos tienen al menos un licenciado/graduado en CCAFD contratado en sus instalaciones. En la figura 8 se observa que, de los graduados/licenciados contratados por los centros, la mayoría desempeña puestos de monitor o instructor (36\%), seguidos por puestos de dirección técnica y coordinación (23\%), y de gerencia y dirección (18\%). Un 15\% y un 8\% desempeñan puestos de entrenador personal y recepción respectivamente.

En los centros de fitness privados de Madrid, García (2011) encontró los siguientes resultados: un 63,9\% de los centros disponían de al menos un licenciado/graduado en CCAFD, y un 35,6\% de los titulados contratados, ocupaban puestos de monitor o instructor.

Parecequehoy en día la función del licenciado/graduado en CCAFD en los centros de fitness se encamina en mayor medida hacia los puestos de monitor o instructor, pero es un dato que debemos matizar, ya que el número de puestos de monitor es mucho mayor cuantitativamente que el resto de puestos disponibles. Se trata sin duda del puesto con más oferta profesional dentro del ámbito del fitness. Según el trabajo de Boned et al. (2015), centrado en el perfil profesional de los trabajadores de los centros de fitness en España, el 11,6\% de los técnicos especialistas en clases colectivas, el $42 \%$ de los entrenadores personales, y el 28,8\% de los técnicos de sala de fitness, tiene la formación de licenciado o graduado en CCAFD. Puig y Viñas (2001) ya apuntaban que el licenciado universitario había encontrado en el mundo del fitness una opción interesante en la que llevar a cabo su carrera profesional, sobre todo cuando parece que salidas habituales para los egresados como son la docencia y el entrenamiento deportivo muestran en la actualidad signos de saturación (Pérez, et al., 2016). El mantenimiento y la salud han supuesto nuevas oportunidades laborales por su virginidad y por la diversidad de aspectos que ofrecen (Viallon, Camy, y Collins, 2003; Pérez, et al. 2016). Los yacimientos laborales vinculados con el mantenimiento y la salud, y con la gestión en el ámbito privado, han incrementado la presencia de graduados en CCAFyD en los últimos años (Pérez, et al., 2016).

Queremos resaltar que nuestras expectativas eran encontrar al menos a un egresado en CCAFD por centro, y bajo nuestro punto de vista hubiera sido deseable encontrar a más titulados formando parte del personal de gerencia, al considerar que su formación es la más adecuada para el desarrollo de funciones de organización y gestión en elámbito del fitness. El trabajo de Boned et al. (2015), mostraba gran heterogeneidad en cuanto a la formación de los miembros del equipo técnico de gerencia. Del personal que desarrollaba funciones de director técnico o coordinador, un 38,8\% tenía formación en CCAFD.

Queremos destacar que, en el citado estudio (Boned et al., 2015), el 19,4\% de los puestos de dirección técnica o coordinación en los centros de fitness estaban cubiertos por personal sin formación oficial, y tampoco tenían formación oficial alguna un 15\% de los técnicos de sala de fitness, un 30\% de los técnicos especialistas en clases colectivas, y un 16\% de los entrenadores personales. También Juan-Llamas (2015) pone de manifiesto en su estudio que casi un $10 \%$ los instructores de clases colectivas no tiene ningún tipo de titulación profesional, aunque todos los instructores sin estudios reglados dicen tener algún tipo de curso de formación no reglada. Nos parece importante recordar también el preocupante dato que arrojaba en su estudio Campos (2013): al describir la formación inicial de los profesionales de la actividad física y del deporte, se obtiene que la mayoría $(57,3 \%)$ de las personas que trabajan en el ámbito concreto del entrenamiento personal no poseía ninguna de las diferentes titulaciones oficiales.

Estos datos evidencian la necesidad de una regulación urgente de la profesión en la totalidad de las comunidades. Consideramos que, por la incidencia de su trabajo en la salud de los clientes, todo el personal que desarrolle labores deportivas en los centros de fitness debería contar con una titulación oficial, o haber sido al menos acreditado por una Agencia Autonómica de Cualificaciones Profesionales.

Consideramos además que la presencia de un titulado universitario en CCAFyD involucrado en tareas de gerencia y dirección, aunque no sea de carácter obligatorio a nivel normativo, debería ser recomendable en la totalidad de los centros de fitness, y que las labores de entrenamiento personal deberían estar realizadas por un egresado en dicha titulación, puesto que estaría realizando funciones de preparación física, propias de su formación.

Sería interesante realizar estudios futuros para comprobar la evolución en la formación de los profesionales de los centros de fitness, sobre todo a raíz de la implementación de la regulación profesional del deporte en las distintas comunidades.

\section{Criterios para la contratación y/o promoción en los centros}

Con referencia a los criterios utilizados para la contratación y/o una posible promoción dentro de la empresa, los contratantes del sector el fitness identifican 4 factores críticos o claves para las distintas ocupaciones, independientemente del puesto a cubrir (Gavín, 2007):

$>$ Las competencias y habilidades específicas para el desempeño del puesto.

$>$ La certificación o titulación que posee.

$>$ La personalidad del trabajador.

$>$ Los años de experiencia en el sector.

En relación a las cualidades más valoradas a la hora de contratar a un empleado, los gerentes/coordinadores de los centros participantes han ordenado las cualidades descritas anteriormente, por orden de preferencia, otorgando una puntuación del 1 al 4 en orden de mayor (1) a menor (4) importancia. A nivel orientativo, se ha catalogado el 1 como «muy importante», el 2 como «bastante importante», el 3 como «importante» y el 4 como «poco importante». Se reflejan en las figuras 9, 10, 11 y 12 las valoraciones de cada uno de estos aspectos por parte de los contratadores.

Podemos ver cómo la experiencia resulta «muy importante» para el $22 \%$ de los gerentes/coordinadores, y «bastante importante» para el $39 \%$. Se trata de la cualidad mejor valorada por la mayoría de los contratadores de entre las ofertadas. De esta forma, el aspecto más relevante a la hora de contratar o promocionar a un trabajador es la experiencia profesional en el sector, seguida de las competencias y habilidades en el puesto. Le siguen la personalidad del trabajador, y el aspecto al que menos valor otorgan de los cuatro tenidos en cuenta en el cuestionario es la formación que poseen. 


\section{Imagen corporativa del centro}

En la figura 13 se observa que el 53\% de los centros encuestados piden al trabajador que adapte su presencia física acorde con la imagen corporativa. Es muy similar el número de centros que exige este requisito del que dejan libremente al profesional escoger su imagen.

Aunque de forma tradicional el sector del fitness ha dado más importancia a las infraestructuras, al equipamiento o la decoración que al factor humano (Boned, et al., 2015), todos ellos elementos relacionados con la imagen, parece que esta tendencia ha cambiado en los últimos años auspiciada por un mercado cada vez más competitivo y unos clientes más exigentes (Gambau, 2011). Disponer de buenos técnicos puede ser un punto importante a tener en cuenta por el gestor deportivo para así fidelizar a sus clientes (Juan-Llamas, 2015), y parece que la tendencia es que otros aspectos quedan supeditados a éste.

\section{Conclusiones}

No hay un perfil claro en cuanto al género del trabajador del ámbito del fitness en la ciudad de Zaragoza, puesto que los porcentajes de hombres y mujeres están muy igualados. La gran mayoría de ellos (91\%) son contratados por la empresa, y su contrato suele ser a tiempo parcial (58\%).

Los centros de trabajo suelen establecer políticas de incentivación de la formación continua, y la forma preferida de hacerlo es favorecer la realización de cursos ajenos a la empresa que suelen suscitar el interés por parte de los trabajadores (64,3\%). Un 57\% de los centros dicen organizar las formaciones en el propio centro. Además, el 21\% dice contar con documentación actualizada y especializada a disposición de sus trabajadores. Los gastos de esta formación continua suelen ser compartidos entre trabajador y empresa (en el 79\% de los centros).

Un 56\% de los centros de fitness participantes afirma contar con un catálogo de funciones y características de cada puesto de trabajo a realizar, y la gran mayoría de los centros cuenta al menos con un egresado en CCAFD contratado. La mayoría de estos titulados cubre funciones de monitor o instructor, aunque en el 23\% de los casos realiza labores de dirección técnica o coordinación, y en el 18\% de los casos, de gerencia y dirección.

La experiencia es valorada por un $22 \%$ de los encuestados como un factor «muy importante» a la hora de realizar la contratación de sus trabajadores, y un 39\% la valora como aspecto «bastante importante». Entre los encuestados prima la experiencia profesional en el sector, seguida de las competencias y habilidades en el puesto, la personalidad del trabajador, y la titulación que poseen, en ese orden, como factores tenidos en cuenta a la hora de contratar a sus profesionales. Dichos trabajadores deben llevar en un 53\% de los casos una indumentaria acorde con la imagen corporativa del centro de trabajo.

Es necesario señalar como una debilidad de nuestro estudio el alto error muestral (18\%). A pesar de haber logrado contactar con la totalidad del universo de estudio, un $9 \%$ de los centros rehusaron participar, y un $56 \%$ de los centros no cumplimentaron la encuesta pese haberla recibido y haber adquirido el compromiso previo de participación. Esto nos hace plantear la necesidad de realizar variaciones en el procedimiento de recogida de información en futuros estudios. Sin duda se obtendría una mayor tasa de respuesta si los cuestionarios se rellenasen a través de una entrevista directa. En todo caso, no fue fácil contactar con muchos de los encuestados por vía telefónica debido a su poco tiempo libre durante su permanencia en el centro de trabajo y su elevada implicación laboral, por lo que estimamos que realizar entrevistas con cada uno de ellos hubiera sido complejo. Quizá ese escaso tiempo libre manifestado haya sido uno de los motivos de la baja tasa de respuesta (35\%), aunque también nos planteamos otras motivaciones como un exceso de celo hacia los datos a proporcionar en el cuestionario, a pesar del anonimato de los mismos. No hay que olvidar la gran competencia existente entre los centros de fitness, agravada en los últimos años.

Vemos necesario seguir realizando trabajos que arrojen luz sobre la situación profesional en el sector del fitness. Su labor es trascendental puesto que se persiguen objetivos relacionados con la salud de los clientes, y porque los recursos humanos se han evidenciado como uno de los puntos fuertes en la satisfacción de los usuarios en los centros deportivos. Se trata de una salida laboral en auge, no regulada en la mayoría de las comunidades autónomas, y escasamente estudiada.

\section{Referencias}

Alves, S. C., da Costa, V.A., Castañer, M., Fernández, J. J., \& Anguera, M. T. (2013). La conducta de los instructores de Fitness: triangulación entre la percepción de los practicantes, auto-percepción de los instructores y conducta observada. Revista de Psicología del Deporte, 22(2), 321-329.

Boned, C.J., Felipe, J.L., Barranco, D., Grimaldi-Puyana, M. \& Crovetto, M. (2015). Perfil profesional de los trabajadores de los centros de fitness en España / Professional Profile of Workers in Spanish Fitness Clubs. Revista Internacional de Medicina y Ciencias de la Actividad Física y el Deporte, 15(58), 195-210.

Campos, A. (2006). Situación profesional de las personas que trabajan en actividad física y deporte en la Comunidad Autónoma de Valencia. Tesis doctoral publicada. Universidad de Valencia, Departamento de Educación Física y Deportiva.

Campos, A., González, M.D., Abella C.P. \& Mestre Sancho, J.A. (2012) Resumen de Situación laboral de los entrenadores personales en la Comunidad Valenciana. Aloma, 30(1), 167-174.

Campos A., González M.D., Pablos C. \& Mestre J. (2013). Situación Sociodemográfica y formativa de los entrenadores personales en la Comunidad Valenciana. Retos. Nuevas tendencias en Educación Física, Deportey Recreación, 24, 57-59.

Consejo Superior de Deportes (2007) Censo Nacional de Instalaciones Deportivas. Madrid: CSD.

Consultora Management Around Sports (2015). Informe global de las instalaciones deportivas españolas 2014. Recuperado de http:// www.cmdsport.com/app/uploads/2015/10/informe-global-de-lasinstalaciones-deportivas-espanolas-2014.pdf

DoYoung, P., Hyungil, K., Teck, K. \& CheeKeng, W. (2010). Perceived Coaching Leadership of Youth Athletes in Singapore. Journal of Sport Behavior, 33(1), 25-41.

Gallardo Pérez, J., \& Campos Izquierdo, A. (2011). Situación profesional de los recursos humanos de la actividad física y el deporte en el municipio de Coslada. Revista Internacional de Medicina y Ciencias de la Actividad Física y el Deporte, 11(43), 440-454.

Gambau, V. (2011). Deporte y empleo en España: dificultades de estudio y de intervención. Revista Española de Educación Física y el Deporte, 394, 13-36.

García, J., Lago, J., \& Fernández, J. (2011). Gestión del tiempo de directores deportivos en centros fitness. Revista Internacional de Medicina y Ciencias de la Actividad Física y el Deporte, 11(44), 674-689.

García, S. (2011). Características de los centros de fitness de titularidad privada de la Comunidad de Madrid. Tesis Doctoral. Madrid: Universidad Europea de Madrid.

Gavín, J. (2007), IDEA Fitness Industry Compensation Survey 2006. IDEA Fitness Journal. Recuperado de http://www.ideafit.com.

Instituto Nacional de las Cualificaciones-Consejo Superior de Deportes. (2008). La Familia Profesional de las Actividades Físicas y Deportivas. Madrid: Ministerio de Educación, Política Social y Deporte. Gobierno de España.

International Health \& Racquet Sports Association. (2007). The State of the Health Club Industry. IHRSA Global Report. Boston: IHRSA.

International Health \& Racquet Sports Association. (2011). The State of the Health Club Industry. IHRSA Global Report. Boston: IHRSA.

International Health \& Racquet Sports Association. (2013). The State of the Health Club Industry. IHRSA Global Report. Boston: IHRSA.

Juan-Llamas, C. (2015). Professional profile of the group classes instructors in Spain. AGONInternational Journal of SportSciences, 5(2), 114-125.

Lagrosen, S. \& Lagrosen, Y. (2007) Exploring service quality in the 
health and fitness industry, Managing Service Quality: An International Journal, 17(1), 41-53.

Lippke, S., Knäuper, B. \& Fuchs, R. (2003). Subjective Theories of Exercise Course Instructors: Causal Attributions for Dropout in Health and Leisure Exercise Programmes. Psychology of Sport and Exercise, 4(2), 155-173.

Loughead, T. M., Colman, M. M. \& Carron, A. V.(2001). Investigating the Mediational Relationship of Leadership Class Cohesion, and Adherence in an Exercise Setting. Small Group Research, 32(5), 558-575.

Loughead, T.M. \& Carron,A. V.(2004). The Mediating Role of Cohesion in the Leader Behavior - Satisfaction Relationship. Psychology of Sport and Exercise, 5, 355-371.

Martínez, J. González, M.D., Jiménez-Beatty, J.E., Graupera, J.L., Martín, M., Campos \& Del Hierro, D., (2009). Los hábitos de actividad física de las mujeres mayores en España. Revista Internacional de Ciencias del Deporte, 5(14), 8193.

Nuviala, A., Tamayo, J. A., Iranzo, J., \& Falcón, D. (2008). Creación, diseño, validación y puesta en práctica de un instrumento de medición de la satisfacción de usuarios de organizaciones que prestan servicios deportivos. Retos. Nuevas tendencias en Educación Física, Deporte y Recreación, 14(2), 10-16.
Pérez, M., Vilanova, A. \& Grimaldi-Puyana, M. (2016). Mercado actual de trabajo de los graduados en Ciencias de laActividad Física y del Deporte: mirada hacia el autoempleo. Journal os Sports Economics \& Management, 6(3), 149-162.

Portes-Junior, M., Lagos, J. A., Domínguez-Riquelme, C. S., \& LópezLópez, R. A. (2014). Formación académica de los instructores de gimnasios de la ciudad de Chillán. Revista Ciencias de la Actividad Física UCM. 15(1), 59-73.

Puig, N. \& Viñas, J. (2001). Mercat de treball i llicenciatura en Educació Física a l'INEF-Catalunya, Barcelona (1980-1997). Barcelona: Diputació de Barcelona, Xarxa de Municipis, Àrea d'Esports.

Telecyl Estudios (2006). Estudio sectorial: Actividades deportivas y sus instalaciones. Principales conclusiones. Estudio realizado por la Fundación Tripartita para la formación en el empleo. Documento sin publicar.

Tracogna, V., Rial-Rebullido, T. (2018). Expectativas y beneficios percibidos del ejercicio hipopresivo por mujeres: una experiencia práctica. Retos. Nuevas tendencias en Educación Física, Deportey Recreación, 34.

Viallon, R., Camy, J., \& Collins, M. (2003). The European integration of a new occupation, the training and education strategies of national professional organizations: the case of the fitness sector in France and the United Kingdom. Managing Leisure, 8(2), 85-96.

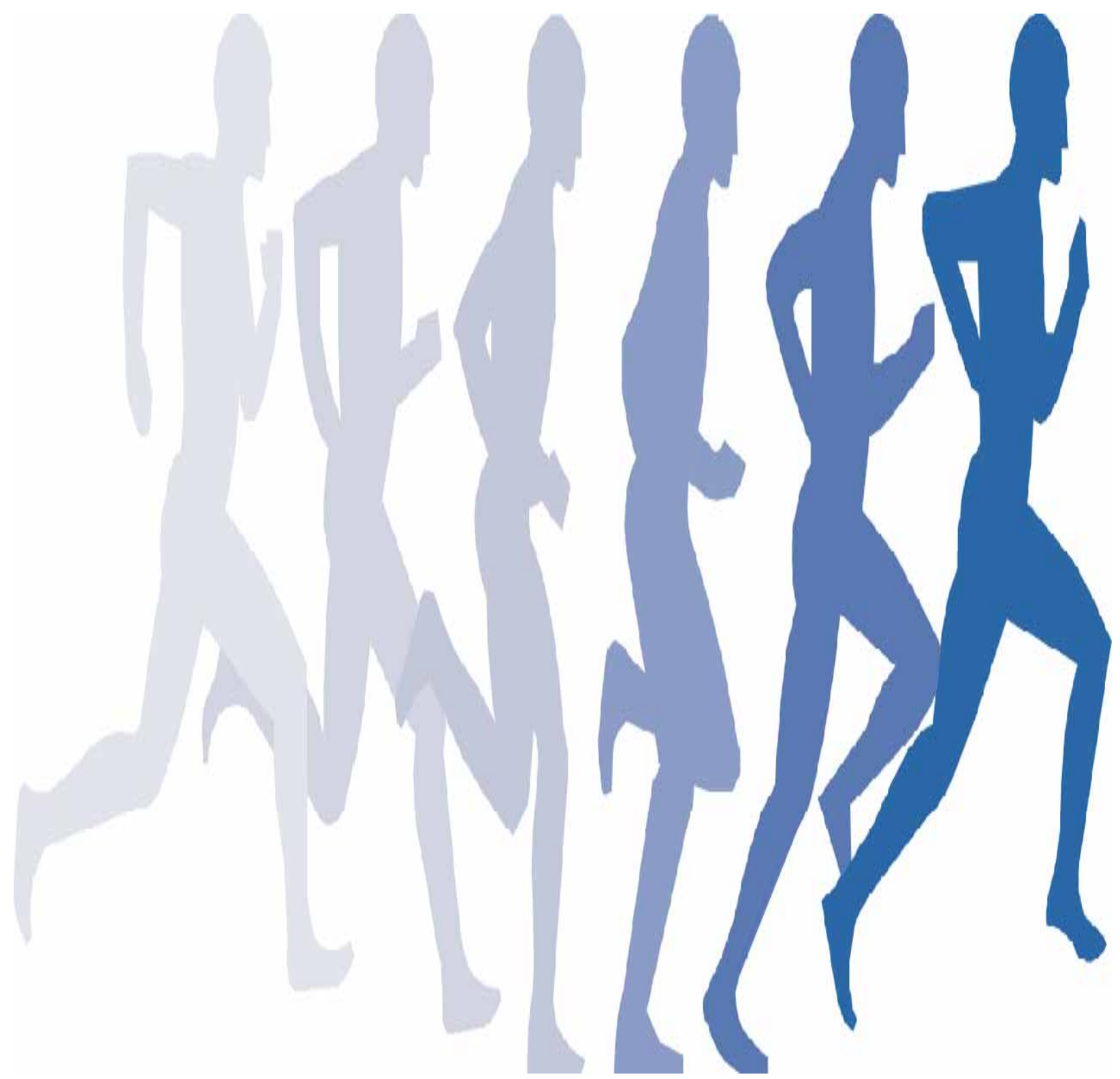

